\title{
Strategies of elite Chinese gymnasts in coping with landing impact from backward somersault
}

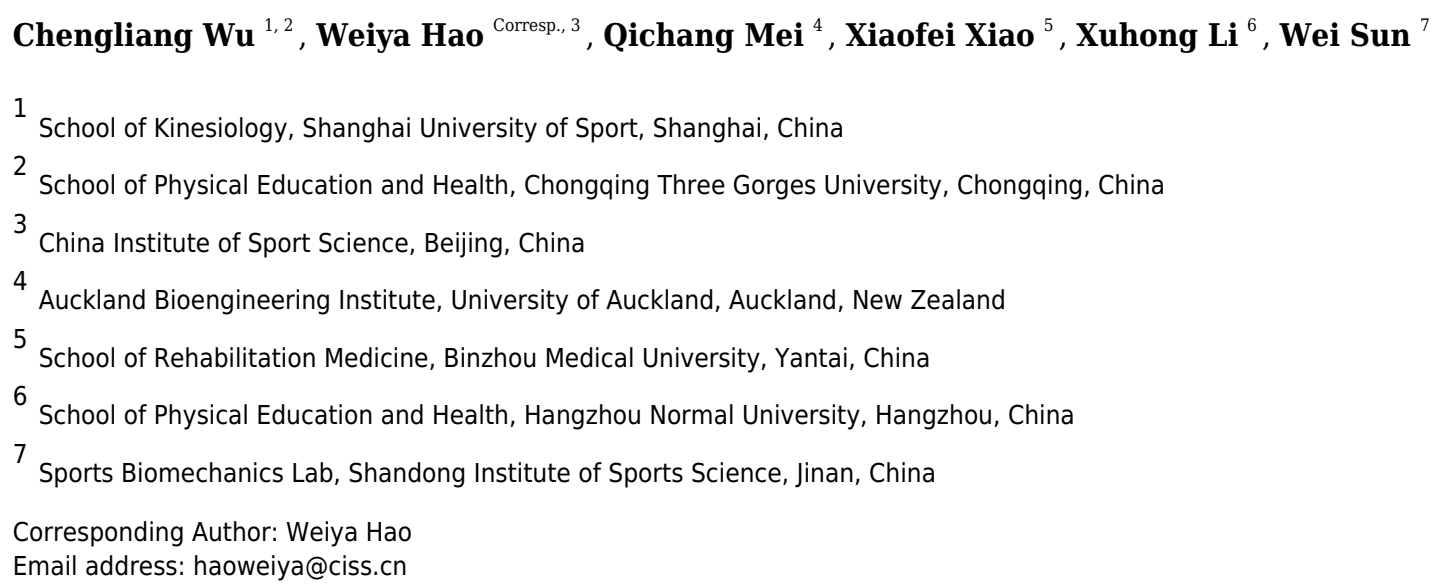

This study aimed to investigate how elite Chinese gymnasts manage the landing impact from a backward somersault. Six international-level male gymnasts performed backward somersault tests with a synchronous collection of kinematics $(250 \mathrm{~Hz})$, ground reaction forces $(1000 \mathrm{~Hz})$, and sur face electromyography $(2000 \mathrm{~Hz})$. A 19-segment human model was developed and lower extremity joints torques were calculated by means of a computer simulation. The angles of the lower extremity joints initially extended and then flexed. These angular velocities of extension continued to decrease and the joint torques changed from extensor to flexor within $100 \mathrm{~ms}$ before touchdown. The angles of the hips, knees, and ankles flexed rapidly by $12^{\circ}, 36^{\circ}$, and $29^{\circ}$, respectively, and the angular velocities of flexion, flexor torque, and electromyography peaked sharply during the initial impact phase of the landing. The angles of the hips, knees, and ankles flexed at approximately $90^{\circ}, 100^{\circ}$, and $80^{\circ}$, respectively. The torques were reversed with the extensor torques, showing a relatively high level of muscle activation during the terminal impact phase of the landing. The results showed that the international-level gymnasts first extended their lower extremity joints, then flexed just before touchdown. They continued flexing actively and rapidly in the initial impact phase and then extended to resist the landing impact and maintain body posture during the terminal impact phase of the landing. The information gained from this study could improve our understanding of the landings of elite gymnasts and assist in injury prevention. 
1 Strategies of elite Chinese gymnasts in coping with landing

2 impact from backward somersault

3 Chengliang $\mathrm{Wu}^{1,2}$, Weiya $\mathrm{Hao}^{3}$, Qichang $\mathrm{Mei}^{4}$, Xiaofei Xiao ${ }^{5}$, Xuhong $\mathrm{Li}^{6}$, Wei Sun ${ }^{7}$

4

$5{ }^{1}$ School of Kinesiology, Shanghai University of Sport, Shanghai, China

6 '2 School of Physical Education and Health, Chongqing Three Gorges University, Chongqing,

7 China

$8{ }^{3}$ China Institute of Sport Science, Beijing, China

$9{ }^{4}$ Auckland Bioengineering Institute, University of Auckland, Auckland, New Zealand

$10{ }^{5}$ School of Rehabilitation Medicine, Binzhou Medical University, Yantai, Shandong, China,

11 6School of Physical Education and Health, Hangzhou Normal University, Hangzhou, Zhejiang,

12 China

$13{ }^{7}$ Sports Biomechanics Lab, Shandong Institute of Sports Science, Jinan, China

14

15 Corresponding Author:

16 Weiya $\mathrm{Hao}^{3}$

17 China Institute of Sport Science, 11 Tiyuguan Road, Beijing, 100061, P. R. China

18 E-mail address: haoweiya@,ciss.cn 
19

20

\section{Abstract}

This study aimed to investigate how elite Chinese gymnasts manage the landing impact from a backward somersault. Six international-level male gymnasts performed backward somersault tests with a synchronous collection of kinematics $(250 \mathrm{~Hz})$, ground reaction forces $(1000 \mathrm{~Hz})$, and surface electromyography $(2000 \mathrm{~Hz})$. A 19-segment human model was developed and lower extremity joints torques were calculated by means of a computer simulation. The angles of the lower extremity joints initially extended and then flexed. These angular velocities of extension continued to decrease and the joint torques changed from extensor to flexor within $100 \mathrm{~ms}$ before touchdown. The angles of the hips, knees, and ankles flexed rapidly by $12^{\circ}, 36^{\circ}$, and $29^{\circ}$, respectively, and the angular velocities of flexion, flexor torque, and electromyography peaked sharply during the initial impact phase of the landing. The angles of the hips, knees, and ankles flexed at approximately $90^{\circ}, 100^{\circ}$, and $80^{\circ}$, respectively. The torques were reversed with the extensor torques, showing a relatively high level of muscle activation during the terminal impact phase of the landing. The results showed that the international-level gymnasts first extended their lower extremity joints, then flexed just before touchdown. They continued flexing actively and rapidly in the initial impact phase and then extended to resist the landing impact and maintain body posture during the terminal impact phase of the landing. The information gained from this study could improve our understanding of the landings of elite gymnasts and assist in injury prevention.

\section{Introduction}

Gymnastics is a popular sport with 50 million participants worldwide (Slater et al., 2015). Each gymnastics routine ends with a landing, and successful landings (without taking a step or falling) are a key factor for motion evaluation. However, there is a high incidence of injury reported in gymnasts while landing, especially to the lower extremity joints (Westermann et al., 2015). A better understanding of the potential injury mechanisms could help prevent certain injuries and change the design of the training schemes, thereby improving performance.

Gymnasts were reported to bear high frequency (over 200 times a week) landing impact loads (Gittoes \& Irwin, 2012), with the peak vertical ground reaction force (vGRF) reaching 7.115.8 times the athlete's body weight (BW) (Slater et al., 2015). This repetition and large GRF 
49 has caused a high rate of lower extremity injuries in gymnasts (Mills, Pain \& Yeadon, 2009). It

50 has also been suggested that there is a correlation between the high injury incidence and the excessive load to the lower extremities of gymnasts (Daly, Bass \& finch, 2001; Wade et al.,

52 2012). Furthermore, the Code of the International Gymnastics Federation (FIG) requires that 53 there be no excessive knee flexion during landing from gymnasts for aesthetic purposes (FIG,

54 2017). Several studies demonstrated that gymnasts produced greater peak vGRF than

55 recreational athletes in drop landings, which are considered the stiff lower extremity landing 56 techniques, with knee flexion less than 90 degrees (Christoforidou et al., 2017; Devita \& Skelly, 57 1992; Seegmiller \& McCaw, 2003). More specifically, this landing pattern increased leg stiffness 58 and is a potential contributing factor for injury (Butler, Crowell \& Davis, 2003). Bradshaw and 59 Hume (2012) investigated the changes in the landings of two gymnasts over a period of eight 60 years. Both gymnasts showed an increased ankle plantar-flexion stiffness by 10.8 and 13.9 $61 \mathrm{kN} / \mathrm{m}$, respectively, with both gymnasts reporting severe pain in one or both heels over this 62 period of time. Furthermore, other contributing factors to injuries in these gymnasts, including 63 internal factors such as anatomical differences, neuromuscular function, strength, and leg 64 stiffness, were observed. External factors, such as the landing of complex tasks, exposure time, 65 the training environment, and varied competition were considered (Bradshaw \& Hume, 2012).

66 Few studies have attempted to explore strategies for reducing the impact force and the 67 incidence of injury. One study found that increasing the flexion of the lower extremity joints 68 during landing could effectively reduce the impact load (Slater et al., 2015). However, this may 69 lead to compensatory muscle and ligament injuries, thus affecting the landing stability, especially 70 during a high-speed landing impact (Bradshaw \& Hume, 2012; Tant, Wilkerson \& Browder, 71 1989). Modifying the material composition of the gymnastics landing mat may reduce the vGRF, 72 but increase the internal load (muscle forces and joint reaction forces) to the lower extremity 73 joints (Mills, Pain \& Yeadon, 2009) and the potential for subtalar and ankle instability during the 74 landing (McNitt-Gray, 2000). It is worth noting that previous studies generally analyzed the 75 whole impact phase of the landing, defined from the initial ground contact to the maximal knee 76 flexion (Christoforidou et al., 2017), the maximal descending height of the center of mass (Caine et al., 2003), or the local minima in the vGRF (McNitt-Gray et al., 2001). Participants in these studies were collegiate students or young athletes who did not participate in international- or 79 national-level gymnastics competitions. Therefore, it is unclear what strategies elite 
80 international-level gymnasts used and whether they adapted initial (from the touchdown to the 81 peak vGRF) and terminal impact-phase (from the peak vGRF return to their body weight) 82 strategies for the flexion/extension of the lower extremity joints before the touchdown of the 83 landing. Moreover, the lower extremity joints approach full extension and then flexion just 84 before touchdown during the flight phase of drop landings (McNitt-Gray, Yokoi \& Millward, 85 1993). However, it is not clear whether the same strategies would be seen before the touchdown of landings from other completed tasks in gymnastics. Using an in-vivo implanted sensor to test the internal load of the human lower extremity would be useful but there are ethical limitations to this methodology. A computer simulation of the human body, however, provides a practical approach to explore the characteristics of body motion and has been widely used to analyze body movement in humans (Pandy, 2001).

The backward somersault (BS) is one of the most basic and common movements for gymnasts for developing difficulty movement and combined motion, and is used frequently in gymnastics training and competitions. The aim of this study was to investigate how elite gymnasts manage the landing impact from a backward somersault and achieve a safe, aesthetic, and stable performance. It is hypothesis that elite gymnasts utilize varied flexion/extension strategies to control their lower extremity joints during the different landing phases (before touchdown, initial, and terminal impact-phases of the landing) of the backward somersault. Therefore, a study of the basic movements would help us understand the characteristics and injury mechanisms of the more complex gymnastics landing of the same type.

\section{Materials \& Methods}

\section{Participants}

Six international-level male gymnasts from the Chinese national team competing in World Cups and/or Championships, with no musculoskeletal injuries for at least six months prior, participated in the study (mean \pm standard deviation (SD) age: $17.3 \pm 1.3$ years, height: $165.7 \pm 5.0 \mathrm{~cm}$, body mass: $57.3 \pm 3.9 \mathrm{~kg}$ ). All participants were familiarized with the procedures in advance and informed consents were signed. The study was approved by the Ethical Advisory Committee of the China Institute of Sport Science (WEI 16-27) in accordance with the regulations set forth by the Declaration of Helsinki.

\section{Procedure}


110 The experiment was conducted in the biomechanics laboratory of the China Institute of Sport 111 Science. A 9-camera Qualisys Oqus motion system (250 Hz, Gothenburg, Sweden) was used to 112 capture the 3D motion data. The standard reflective markers (diameter: $16 \mathrm{~mm}$ ) were placed at 113 the head, cervical vertebrae (CV7), scapula-inferior angle, thoracic vertebrae (TV10), shoulder, 114 elbow, wrist, anterior superior iliac spine, posterior superior iliac spine, knee, ankle, metatarsal115 phalangeal joints, heel, and toes on both sides of the body (Fig. 1A-C). The marker placements 116 were referenced from the CAST full body marker set (Sint, 2007). A Kistler force plate $117(400 \times 600 \mathrm{~mm})$, located beneath a landing mat $(5 \mathrm{~cm}$ thick), was used to collect vGRF data $(1000$ $118 \mathrm{~Hz}$ ) and was surrounded by an ethylene-vinyl acetate insole mat. The vGRF was reported to 119 decrease by $5 \%$ on the force plate when the landing mats were up to $12 \mathrm{~cm}$ thick (McNitt-Gray et 120 al., 2001). Surface muscle activity signals were recorded (2000 Hz, Bagnoli 8 Desktop 121 electromyography (EMG) System; Delsys Inc, Boston, MA) from the rectus femoris (RF), biceps 122 femoris (BF), tibialis anterior (TA), and lateral gastrocnemius (LG) on the two lower extremities 123 of the gymnasts as per the SENIAM guidelines (Hermens et al., 1999). The Qualisys Oqus 124 motion capture system, the Kistler force plate, and the surface EMG system were all 125 synchronized during the data collection.

126 The gymnasts completed an initial warm-up exercise (15 min of jogging, jumping, and 127 stretching) and then each participant performed three successful trials of BS without taking a step 128 or hop. The BS was initiated by jumping from the ground next to the force plate with bare feet 129 (Fig. 2). The best trial for each participant was chosen by two national-level judges using the 130 Code of Points of FIG for further analysis.

\section{Experiment data reduction and analysis}

132 The 3D motion data was processed using Qualisys Track Manager Software, following a $10 \mathrm{~Hz}$ 133 low-pass cut-off filter (Slater et al., 2015). The joint angles with its angular velocity were 134 calculated between two lines in space based on three-dimension trajectories. The vGRFs were 135 filtered using a low-pass cut-off at $50 \mathrm{~Hz}$ (Slater et al., 2015). The peak vGRFs were normalized 136 with the body weight of each gymnast. The touchdown was identified as the first frame when 137 vGRF exceeded $10 \mathrm{~N}$ (Christoforidou et al., 2017). Raw EMG signals were fully wave rectified 138 and band-pass filtered by 10-400Hz (van Dieen et al., 2009).

139 The pre-activation phase (T0) was defined as the $100 \mathrm{~ms}$ duration before touchdown (Komi 140 \& Bosco, 1987). The EMG processing was conducted in the pre-activated and two impact phases 
141 of the landing (T1: initial impact-phase, from contact to the peak vGRF; T2: terminal impact-

142 phase, from the peak vGRF return to their body weight) (Fig. 2). The EMG signals were

143 normalized by the peak EMG of each trial. Antagonist-agonist co-activation was calculated as

144 TA to LG normalized EMG for ankle, and BF to RF normalized EMG for knee (Ruan \& Li,

145 2010). All of the experimental data for the gymnasts were averaged within $20 \mathrm{~ms}$ intervals and

146 then the data was reported as mean \pm SD using descriptive statistics.

147 Computer simulation and validation

148 The experimental findings from the elite gymnasts showed a low discrete degree, with the mean 149 standard error within six participants of 0.6 BW (peak vGRF), $2.7 \mathrm{~ms}$ (time to peak vGRF) and 150 ranging from $2^{\circ}$ to $10^{\circ}$ in the lower extremity angles. One gymnast with intermediate experiment 151 results was chosen for modeling and simulation.

152 LifeMod (LifeModeler, Inc. San Clemente, CA) is an advanced multi-body computer 153 simulation software system commonly used in human movement simulation with ADAMS 154 (Automatic Dynamic Analysis of Mechanical Systems) as the dynamics modeling engine. The 155 GeBod database (BRG.LifeMOD ${ }^{\mathrm{TM}}$ ) was used to develop a 19-segment and 50 DOF (Degree of 156 Freedom) rigid-body model based on age (17 years), weight (63 kg), and height (1.68 $\mathrm{m})$ data 157 from the selected gymnast. The model consisted of the head, neck, upper torso, central torso, 158 lower torso, scapulas, upper arms, lower arms, hands, upper legs, lower legs, and feet (Fig. 1D) 159 (Serveto et al., 2010). A model of the gymnastics matting with a dimension of $2 \times 2 \times 0.05 \mathrm{~m}$ 160 (length $\times$ width $\times$ height) was developed using MSC.ADAMS (MSC Software Corp. acronym 161 of Automated Dynamic Analysis of Mechanical Systems) software. The basis for the mechanical 162 properties of the landing mat was obtained by an optimization algorithm. The model was then 163 validated by coefficients of multiple correlations (CMC) between the simulation and actual 164 results, with the specific algorithm described in detail in our previous study (Xiao et al., 2017). 165 After verifying the reliability of the model, the joint torques of the hip, knee, and ankle joints 166 were conducted using computer simulation.

\section{Results}

168 The measured angles of the hip, knee, and ankle joints were first extended (mean $11^{\circ}, 10^{\circ}$, and $1696^{\circ}$, respectively) and then flexed during T0 in the six gymnasts (Fig. 3A-C). The corresponding 170 joint angles rapidly flexed by $2^{\circ}, 36^{\circ}$, and $29^{\circ}$ during $\mathrm{T} 1$ and maintained at around $90^{\circ}, 100^{\circ}$, and 
$17180^{\circ}$ during $\mathrm{T} 2$, respectively. The angular velocities of extension continued to decrease during $\mathrm{T} 0$, 172 and the angular velocities of flexion reached their peaks during T1 and gradually approached 173 zero during T2 (Fig. 4A-C). The eight muscles in the bilateral lower extremity were pre-activated 174 during T0 (Fig. 5A-D). The EMG amplitude of most muscles increased from T0 to T1, and 175 reached their maximum near the peak vGRF. They still maintained a high-level of activation 176 during $\mathrm{T} 2$.

177 The coefficient of multiple correlations (CMC) between the measured lower extremity joint 178 angles and the simulation result were calculated for model validation, with the left knee 179 (CMC=0.95), right knee ( $\mathrm{CMC}=0.93)$, and left and right ankles ( $\mathrm{CMC}=0.85)$ (Fig. 6A, B). The 180 CMCs greater than 0.75 indicated good correlations (Collins et al., 2009). The difference 181 between the simulated (11.9 BW) and measured peak vGRF (12.5 BW) was 4.6\%, which was 182 less than $10 \%$ and was considered to be an accurate representation (Mark et al., 2006).

183 Therefore, these results could be used to validate the model. The angles and angular velocities of 184 the lower extremity joints were consistent with the results of Fig. 3A-C and Fig. 4A-C, 185 respectively (Fig. 6A-D). The torques of the lower extremity joints were initially dominated by extensor during T0 (plantar flexor for the ankle) (Fig. 6E, F), and reversed to flexor, reaching their maximum (dorsiflexion for the ankle) during T1. The torques quickly reversed again to the extensor and reached their peak during T2 (plantar flexor for the ankle). The torque of the knee joints reached their maximum and in the ankle joints the torques remained slight.

\section{Discussion}

191 From the best of our knowledge, this is the first investigation to reveal the flexion/extension 192 strategies of the lower extremity joints from flight to the initial and terminal impact-phases of 193 backward somersault landings in elite gymnasts. The study quantified the kinematics, kinetics, 194 and muscle activation characteristics of each phase of the backward somersault in elite gymnasts. 195 The findings could enhance our understanding of the gymnastics landing.

The lower extremity joints of the gymnasts first extended and then flexed actively during 197 the preparation phase for the touchdown (T0). Increased angles of the lower extremity joints were conducive to the body's extension, which may contribute to increasing the moment of 199 inertia of the body. As the moment of inertia increases, the gymnasts can reduce their angular velocity in preparation for touchdown (McNitt-Gray, 2000). It was noteworthy that the angular velocities of the knee and ankle joints changed from extension to flexion before touchdown. The 
202 lower extremity joints actively flexed, which was first seen in the ankle joint, followed by the 203 knee and hip joints. Gittoes et al. (2011) suggested that the female gymnasts' knees and ankles 204 were also flexing at the touchdown of the backward rotating pike and tuck dismounts, but there 205 was no information about whether the joints flexed before touchdown. Furthermore, the 206 antagonist and agonistic muscles of the lower extremity joints were pre-activated, which may 207 play an important role in regulating the lower extremity stiffness (Christoforidou et al., 2017). 208 Muscle contraction is an essential factor for producing lower extremity joint torque in the flight 209 before landing. The lower extremity joints experienced the process from extension to flexion 210 because these torques changed from extensor to flexor. Previous studies have focused on the 211 torque of the lower extremity joints after touchdown (McNitt-Gray et al., 2001; Mills, Pain \& 212 Yeadon, 2009; Verniba et al., 2017), but there is little knowledge about the torque of the joints in 213 the pre-landing phase. Our results indicated that in preparation for the landing, the lower 214 extremity joints of the gymnast would first be extended and then actively flexed just before 215 touchdown.

216 During the initial impact-phase of the landing (T1), the lower extremity joints continue 217 flexing actively and rapidly. T1 has a very short duration $(22.8 \pm 6.7 \mathrm{~ms})$, which was consistent 218 with the results of a previous study (Slater et al., 2015) where the angles of the lower extremity 219 joints flexed rapidly. Furthermore, the angular velocities of the hip joints changed from 220 extension to flexion and the angular velocities of the knee and ankle joints further flexed and 221 reached their peak values. It is significant to note that the peak angular velocities of the ankle 222 joint occurred before touchdown, but the peak angular velocities of the knee joint occurred 223 before the peak of the VGRF and the peak angular velocities of the hip joint occurred near the 224 peak vGRF. They reached peak values successively, which may be because the ankle is the 225 initial interface with the ground, and the hip is the proximal joint, which properly reflects the 226 synergy of multiple joints (Gittoes et al., 2011). McNitt-Gray et al. (2001) suggested that only

227 the flexor torques of the hip joints were generated in a short time (about $20 \mathrm{~ms}$ ) after touchdown 228 and the other joints of the lower extremities maintain extensor torques during the landing.

229 However, our results confirmed that all torques of the lower extremity joints were flexor torques 230 in T1. This difference in lower extremity joint torques might be due to the landing being 231 performed by different levels of participants (collegiate male gymnasts in their study against the 232 international-level gymnast in this study). Therefore, the authors speculated that the participants 
233 (international-level gymnasts) in this study would represent an altered landing control strategy to 234 help improve performance. Additionally, the results of muscle activation showed that the EMG 235 amplitudes of the antagonistic and agonistic muscles of the lower extremity joints were close to 236 reaching their peak values synchronously, and therefore this regulated the lower extremity 237 stiffness to accommodate the rapidly increased vGRF (Kramer, 2012). A previous study 238 indicated that the activity levels of the lower extremity muscles positively correlated with the 239 peak vGRF during the landing absorption phase, which contributed to absorbing the impact of 240 the landing and preventing injury to the lower extremities (Iida et al., 2011). Therefore, in order 241 to quickly absorb the landing impact and prevent injuries during T1, the authors suggest that the 242 lower extremity joints of gymnasts may flex continuously and actively, while increasing the 243 activity levels of the lower extremity muscles.

244 During the terminal impact-phase of the landing (T2), the gymnasts in this study began to 245 actively extend their lower extremity joints to resist the impact force and maintain body posture. 246 The angles and angular velocities of the lower extremity joints flexed minutely. Finally, the 247 lower extremity joints stabilized and maintained a small flexion, thus these gymnasts met the 248 Code of Points of FIG, which requires that the joints should not be excessively flexed (FIG, 249 2017). Furthermore, the EMG of the lower extremity muscles remained at a high level of 250 activation, and the extensor torques of the lower extremity joints (plantar flexion torque of ankle) 251 increased simultaneously, reaching their peak values during T2. Therefore, accomplishing the 252 landing task with a small flexion of the lower extremity joints may allow the gymnasts to 253 generate the joint torques needed to compensate for the large vGRF. Appropriate muscle length 254 may be the key to producing greater joint torques (McNitt-Gray, 2000). Marchetti et al. (2016) 255 found that the highest overall muscle activation of the lower extremities was generated at a $90^{\circ}$ 256 knee joint angle during a back squat, which was close to the knee angles of this study during T2.

Devita and Skelly (1992) suggested that a stiff landing in which there is less than 90 degrees of knee flexion had larger GRFs than a soft landing; the ankle plantar flexors produced a larger torque, and the ankle muscles absorbed a greater amount of the impact forces upon landing. The ankle is more likely to be injured in this condition, and this was consistent with the findings of an 261 epidemiological investigation (Kerr et al., 2015). The study indicated that the gymnasts first extend their lower extremity joints before touchdown, then flex actively and rapidly in anticipation of the upcoming touchdown and the initial impact phase, and again extend during 
264 the terminal impact phase of the landing. These landing strategies could effectively mitigate 265 some of the landing impact and are conducive to injury prevention.

266 There are a few limitations in this study. First, this study is limited to a population of 267 international-level male gymnasts, but we acknowledge that there might be differences in lower 268 extremity kinematics and kinetics between different genders during their landings (Haines et al., 269 2011). Secondly, the sample size (six international-level gymnasts) of this study is limited and 270 the authors did not consider more gymnasts of different levels, which was the original intent in 271 order to control variables and reveal the landing strategies primarily in international-level 272 gymnasts. However future studies should include male and female gymnasts of different levels.

\section{Conclusions}

274 The study quantified the lower extremity kinematics, kinetics, and muscle activation of 275 international-level gymnasts during backward somersault landings. Gymnasts first extend their 276 lower extremity joints to increase the moment of inertia, thus better reducing the body's angular 277 velocity before touchdown. The lower extremity joints flex actively just before touchdown, 278 continue flexing actively and rapidly in the initial impact phase, and then extend to resist the 279 impact force and maintain body posture during the terminal impact phase of the landing. This is 280 the first study to investigate altered flexion/extension strategies of the lower extremity joints 281 during different phases of the landing in gymnastics, thereby having the potential to expand the 282 current understanding of the landing process of gymnastics and to aid in the prevention of 283 injuries.

\section{4}

285

286

287

288

289

290

291

292

293

294

295

296

297

\section{Acknowledgements}

The authors are thankful to the Chinese national gymnastics team for their valuable collaboration.

\section{References}

Bradshaw EJ, Hume PA. 2012. Biomechanical approaches to identify and quantify injury mechanisms and risk factors in women's artistic gymnastics. Sports biomechanics 11:324-341 DOI 10.1080/14763141.2011.650186

Butler RJ, Crowell HP, Davis IM. 2003. Lower extremity stiffness: implications for performance and injury. Clinical Biomechanics 18:511-517 DOI 10.1016/s0268-0033(03)00071-8

Caine D, Knutzen K, Howe W, Keeler L, Sheppard L, Henrichs D, Fast J. 2003. A three-year epidemiological study of injuries affecting young female gymnasts. Physical Therapy in Sport 4:10-23 DOI 10.1016/s1466-853x(02)00070-6

Christoforidou A, Patikas DA, Bassa E, Paraschos I, Lazaridis S, Christoforidis C, Kotzamanidis C. 
312

313

314

315

316

317

318

319

320

321

322

323

324

325

326

327

328

329

330

331

332

333

334

335

336

337

338

339

340

341

2017. Landing from different heights: Biomechanical and neuromuscular strategies in trained gymnasts and untrained prepubescent girls. Journal of Electromyography and Kinesiology 32:1-8 DOI 10.1016/j.jelekin.2016.11.003

Collins TD, Ghoussayni SN, Ewins DJ, Kent JA. 2009. A six degrees-of-freedom marker set for gait analysis: repeatability and comparison with a modified Helen Hayes set. Gait Posture 30: 173180DOI 10.1016/j.gaitpost.2009.04.004

Daly RM, Bass, SL, Finch CF. 2001. Balancing the risk of injury to gymnasts: how effective are the counter measures. British Journal of Sports Medicine 35:8-20 DOI 10.1136/bjsm.35.1.8

Devita P, Skelly WA. 1992. Effect of landing stiffness on joint kinetics and energetics in the lower extremity. Medicine \& Science in Sports \& Exercise 24:108-115 DOI 10.1249/00005768-199201000-00018

FIG. 2017. 2017 Code of Points - Men's artistic gymnastics.Lyss: Fe'de'ration Internationale De Gymnastique.

Gittoes MJR, Irwin G. 2012. Biomechanical approaches to understanding the potentially injurious demands of gymnastic-style impact landings. Sports Medicine, Arthroscopy, Rehabilitation, Therapy \& Technology 4:4 DOI 10.1186/1758-2555-4-4

Gittoes MJR, Irwin G, Mullineaux DR, Kerwin DG. 2011. Whole-body and multi-joint kinematic control strategy variability during backward rotating dismounts from beam. Journal of Sports Sciences 29:1051-1058 DOI 10.1080/02640414.2011.576690

Haines TL McBride JM, Triplett NT, Skinner JW, Fairbrother KR, Kirby TJ. 2011. A comparison of men's and women's strength to body mass ratio and varus/valgus knee angle during jump landings. Journal of Sports Sciences 29:1435-1442 DOI 10.1080/02640414.2011.599039

Hermens H FB, Merletti R, Stegeman D, Blok J, Rau G, Disselhorst-Klug C, Hägg G. 1999. European Recommendations for Surface Electromyography. Roessingh Research and Development, Enshede, The Netherlands

Iida Y, Kanehisa H, Inaba Y, Nakazawa K. 2011. Activity modulations of trunk and lower limb muscles during impact-absorbing landing. Journal of Electromyography and Kinesiology 21:602609

DOI 10.1016/j.jelekin.2011.04.001

Komi PV, Bosco C. 1987. Utilization of stored elastic energy in the leg extensor muscles by men and women. Medicne and Science in Sports 10:261-265 DOI 10.1007/BF00423113

Kramer A, Ritzmann R, Gruber M, Gollhofer A. 2012. Leg stiffness can be maintained during reactive hopping despite modified acceleration conditions. Journal of Biomechanics 45:1816-1822 DOI 10.1016/j.jbiomech.2012.04.014

Marchetti PH, Silva JJ, Schoenfeld BJ, Nardi PSM, Pecoraro SL, Greve JMD, Hartigan E. 2016. Muscle Activation Differs between Three Different Knee Joint-Angle Positions during a Maximal Isometric Back Squat Exercise. Journal of Sports Medicine (Hindawi Publ corp) 2016:3846123 DOI 10.1155/2016/3846123

Mark A. King CW, Yeadon MR. 2006. Evaluation of a torque-driven model of jumping for height. Journal of Applied Biomechanics 22:264-274 DOI 10.1123/jab.22.4.264

McNitt-Gray JL. 2000. Musculoskeletal Loading During Landing. In: Zatsiorsky VM, ed. Biomechanics in sport-Performance enhancement and injury prevention. Oxford: Blackwell Science Ltd, 523550 .

McNitt-Gray JL, Hester DME, Mathiyakom W, Munkasy BA. 2001. Mechanical demand and 
342

343

344

345

346

347

348

349

350

351

352

353

354

355

356

357

358

359

360

361

362

363

364

365

366

367

368

369

370

371

372

373

374

375

376

377

378

379

380

multijoint control during landing depend on orientation of the body segments relative to the reaction force. Journal of Biomechanics 34: 1471-1482 DOI 10.1016/s0021-9290(01)00110-5

McNitt-Gray JL, Yokoi T, Millward C. 1993. Landing Strategy Adjustments Made by Female Gymnasts in Response to Drop Height and Mat Composition. Journal of Applied Biomechanics 9:173-190.

Mills C, Pain MTG, Yeadon MR. 2009. Reducing ground reaction forces in gymnastics' landings may increase internal loading. Journal of Biomechanics 42:671-678

DOI 10.1016/j.jbiomech.2009.01.019

Pandy MG. 2001. Computer modeling and simulation of human movement. Annual Review of Biomedical Engineering. 3, 245-273 DOI: 10.1146/annurev.bioeng.3.1.245

Seegmiller JG, McCaw ST. 2003. Ground Reaction Forces Among Gymnasts and Recreati Athletes in Drop Landings. Journal of Athletic Training 38:311-413 DOI 10.1152/japplphysiol.00406.2003

Serveto S, Barre S, Kobus JM, Mariot JP. 2010. A three-dimensional model of the boat-oars-rower system using ADAMS and LifeMOD commercial software. Journal of Sports Engineering and Technology 224:75-88 DOI 10.1243/17543371 jset42

Sint JSV . 2007. Color Atlas of Skeletal Landmark Definitions. Guidelines for Reproducible Manual and Virtual Palpations. Edinburgh: Churchill Livingstone, 29-175.

Slater A, Campbell A, Smith A, Straker L. 2015. Greater lower limb flexion in gymnastic landings is associated with reduced landing force: a repeated measures study. Sports Biomechanics 14:45-56 DOI 10.1080/14763141.2015.1029514

Tant CL, Wilkerson JD, Browder KD. 1989. Technique comparisons between hard and soft landings of young female gymnasts.Journal of Biomechanics22:1088 DOI 10.1016/0021-9290(89)90478-8

van Dieen JH, Westebring-van der Putten EP, Kingma I, de Looze MP. 2009. Low-level activity of the trunk extensor muscles causes electromyographic manifestations of fatigue in absence of decreased oxygenation. J Electromyogrography and Kinesiology, 19, 398-406

Doi:10.1016/j.jelekin.2007.11.010

Verniba D, Vescovi JD, Hood DA, Gage WH. 2017. The analysis of knee joint loading during drop landing from different heights and under different instruction sets in healthy males. Sports Medicine-Open 3:6 DOI 10.1186/s40798-016-0072-x

Wade M, Campbell A, Smith A, Norcott J, O'Sullivan P. 2012. Investigation of Spinal Posture Signatures and Ground Reaction Forces During Landing in Elite Female Gymnasts. Journal of Applied Biomechanics 28:677-686 DOI 10.1123/jab.28.6.677

Westermann RW, Giblin M, Vaske A, Grosso K, Wolf BR. 2015. Evaluation of Men's and Women's Gymnastics Injuries: A 10-Year Observational Study. Sports Health 7:161-165 DOI 10.1177/1941738114559705

Xiao X, Hao W, Li X, Wan B, Shan G. 2017. The influence of landing mat composition on ankle injury risk during a gymnastic landing:a biomechanical quantification. Acta of Bioengineering and Biomechanics 19:105-113. DOI10.5277/abb-00583-2016-02

Peer] reviewing PDF | (2019:04:36579:2:0:NEW 6 Sep 2019) 


\section{Figure 1}

Location of retro-reflective markers and EMG sensors on the gymnasts ( $A, B$, and $C), 19$ segments human model generated by GEBOD in LifeMOD ${ }^{T M}$ (D).

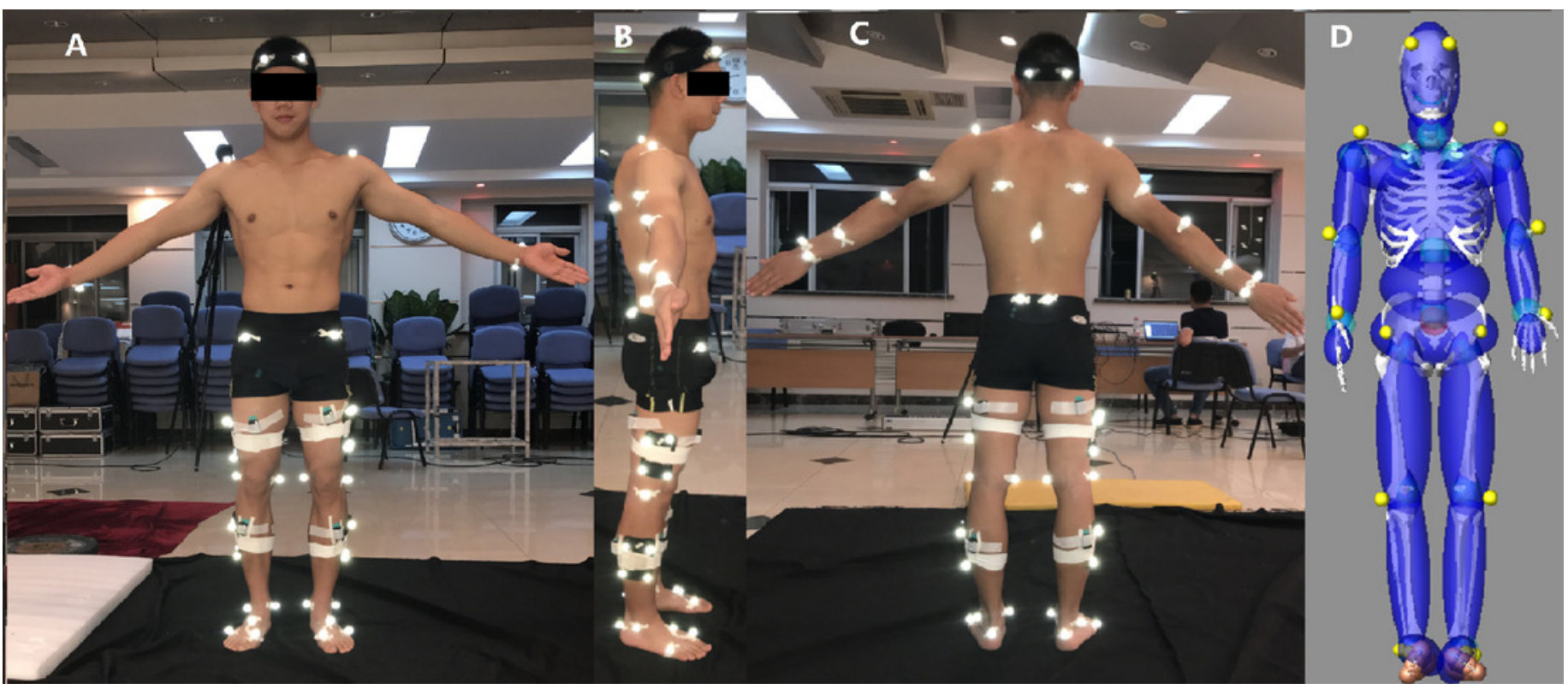


Figure 2

The demonstration of backward somersault landing.

T0: The pre-activation phase was defined as 100 ms preceding ground contact; T1: initial impact-phase, from the first touchdown to the peak vertical ground reaction force (VGRF); T2: terminal impact-phase, from the peak VGRF to the VGRF equaling to body weight.

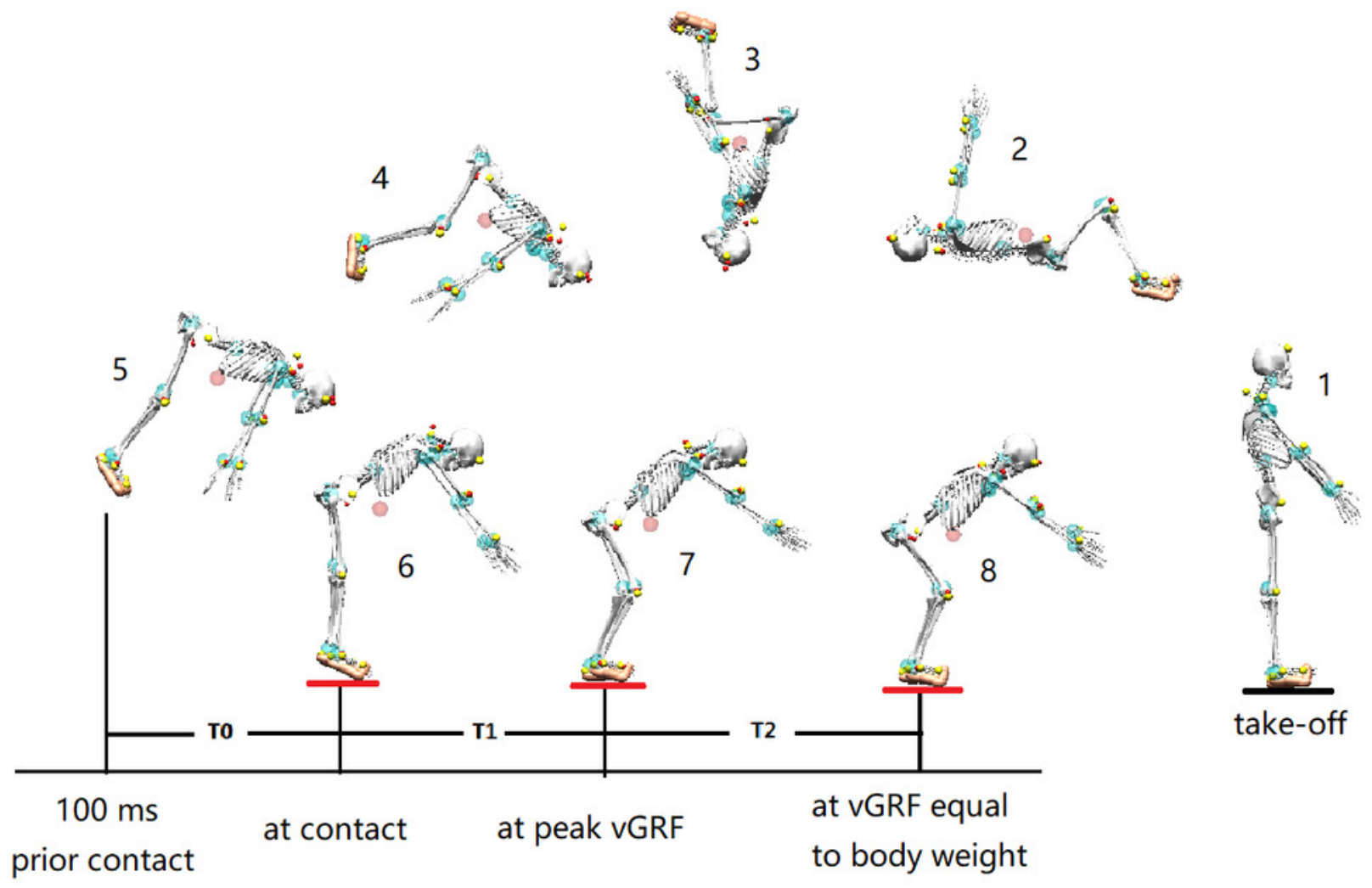




\section{Figure 3}

Average integrated (20 ms bins) angles of lower extremity joints (A, B, and C) and vertical ground reaction force (vGRF) (D) during backward somersault landing $(n=6)$.

The whole landing process was divided into three phases by solid vertical lines. T0: The preactivation phase was defined as $100 \mathrm{~ms}$ preceding ground contact; T1: initial impact-phase, from the first touchdown to the peak VGRF; T2: terminal impact-phase, from the peak VGRF to the VGRF equaling to body weight (Mean: solid vertical line, standard deviation: dotted vertical line). 


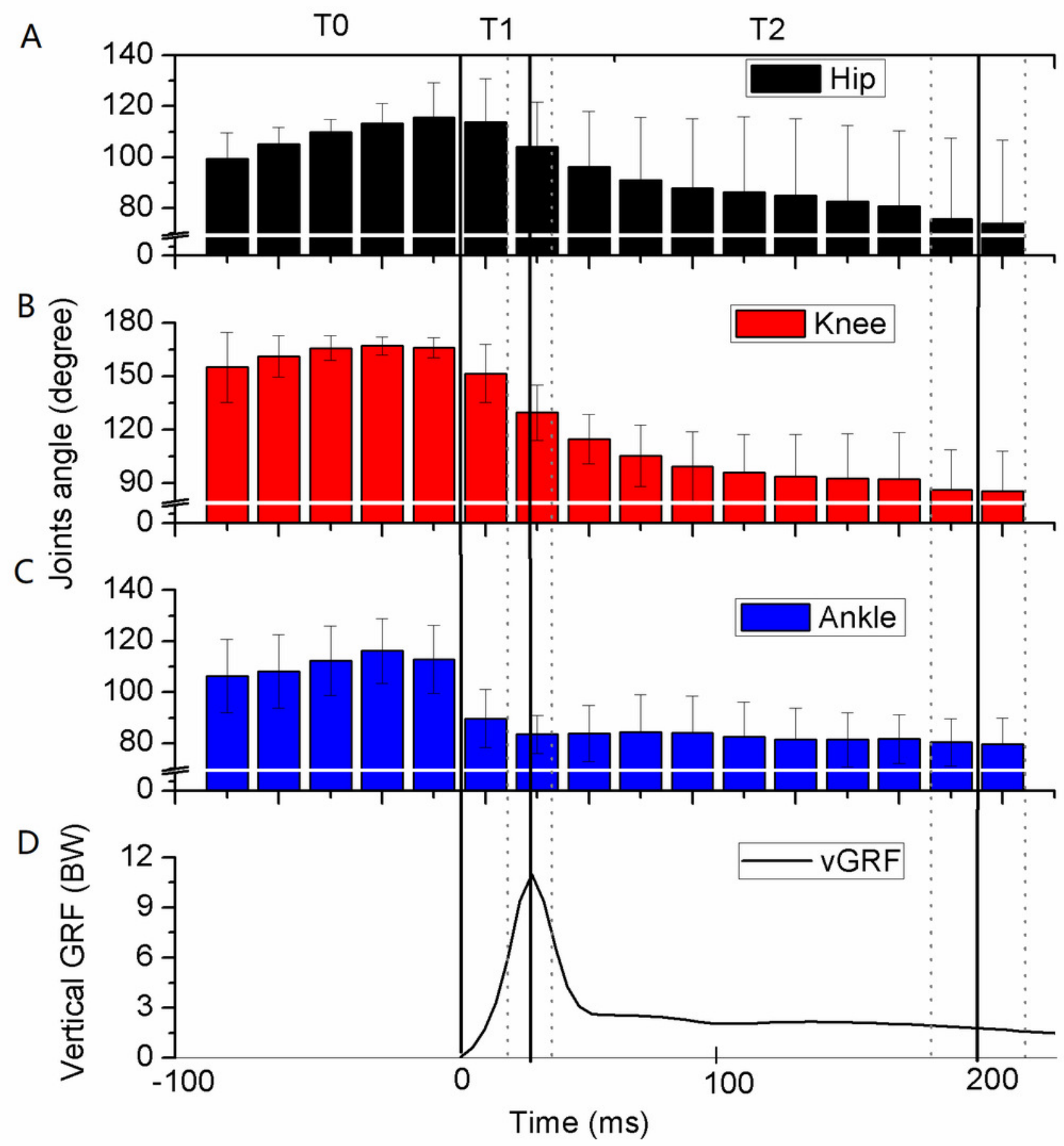




\section{Figure 4}

Average integrated (20 ms bins) angular velocities of lower extremity joints during backward somersault landing $(A, B$, and $C)(n=6)$.

The solid arrow indicates that the acceleration is negatively increasing, and the dotted arrow indicates that the acceleration is negatively decreasing. The whole landing process was divided into three phases by solid vertical lines. T0: The pre-activation phase was defined as 100 ms preceding ground contact; T1: initial impact-phase, from the first touchdown to the peak vertical ground reaction force (VGRF); T2: terminal impact-phase, from the peak VGRF to the VGRF equaling to body weight (Mean: solid vertical line, standard deviation: dotted vertical line). 


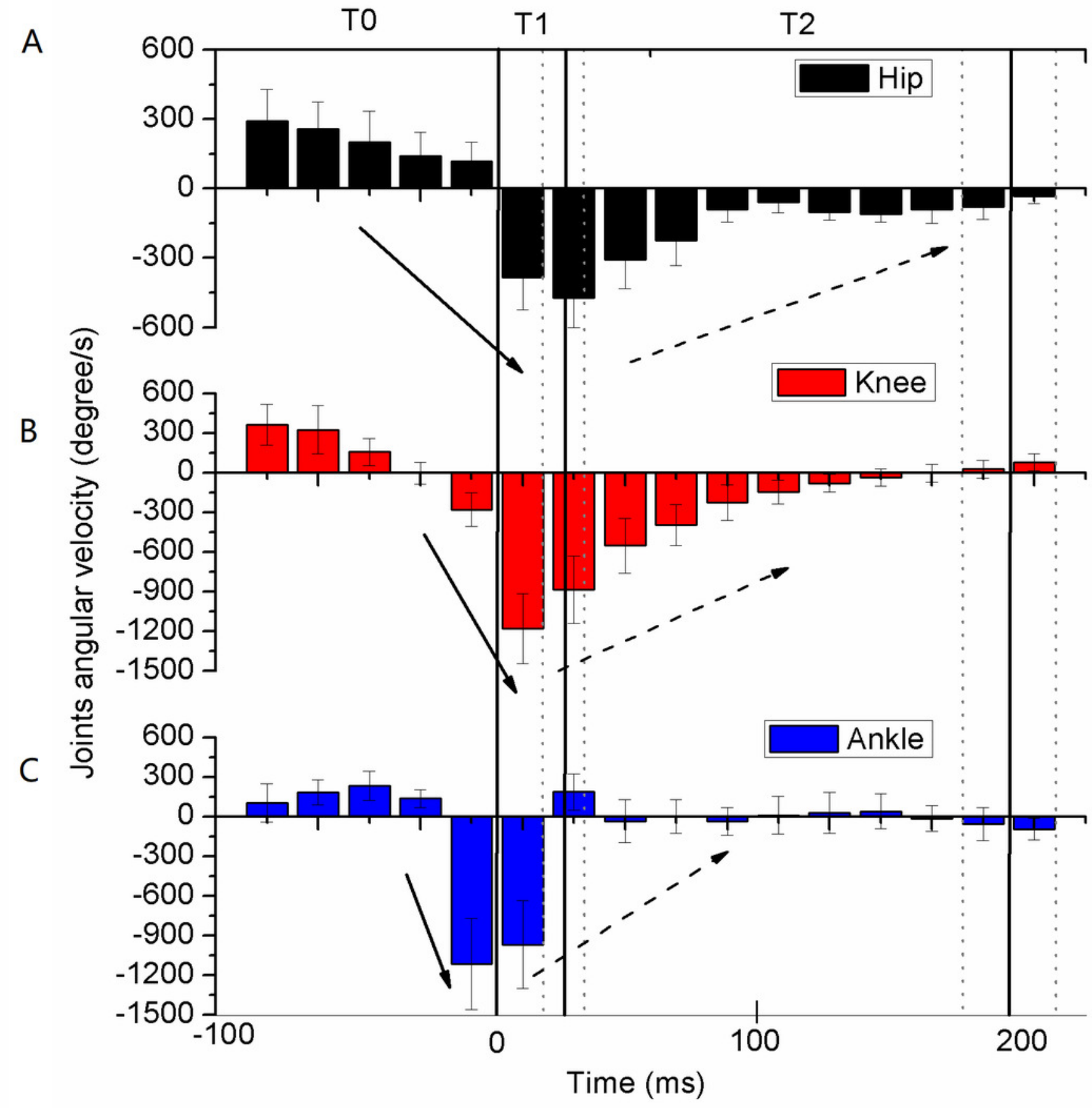




\section{Figure 5}

Average integrated (20 ms bins) coactivation of root mean square of normalized EMG $\left(E M G_{R M S}\right)(n=6)$.

Positive $E M G_{R M S}$ means antagonist of the joint, and negative $E M G_{R M S}$ means the agonist of the joint. The EMGs were normalized to the peak EMG during the landing phase. RF: rectus femoris, BF: biceps femoris, TA: tibialis anterior, LG: lateral gastrocnemius. The coactivation was defined as the TA to LG EMG for the ankle (A,C), and the BF to RF EMG for the knee (B, D). The whole landing process was divided into three phases by solid vertical lines. T0: The pre-activation phase was defined as 100 ms preceding ground contact; T1: initial impactphase, from the first touchdown to the peak vertical ground reaction force (vGRF); T2: terminal impact-phase, from the peak VGRF to the vGRF equaling to body weight (Mean: solid vertical line, standard deviation: dotted vertical line).
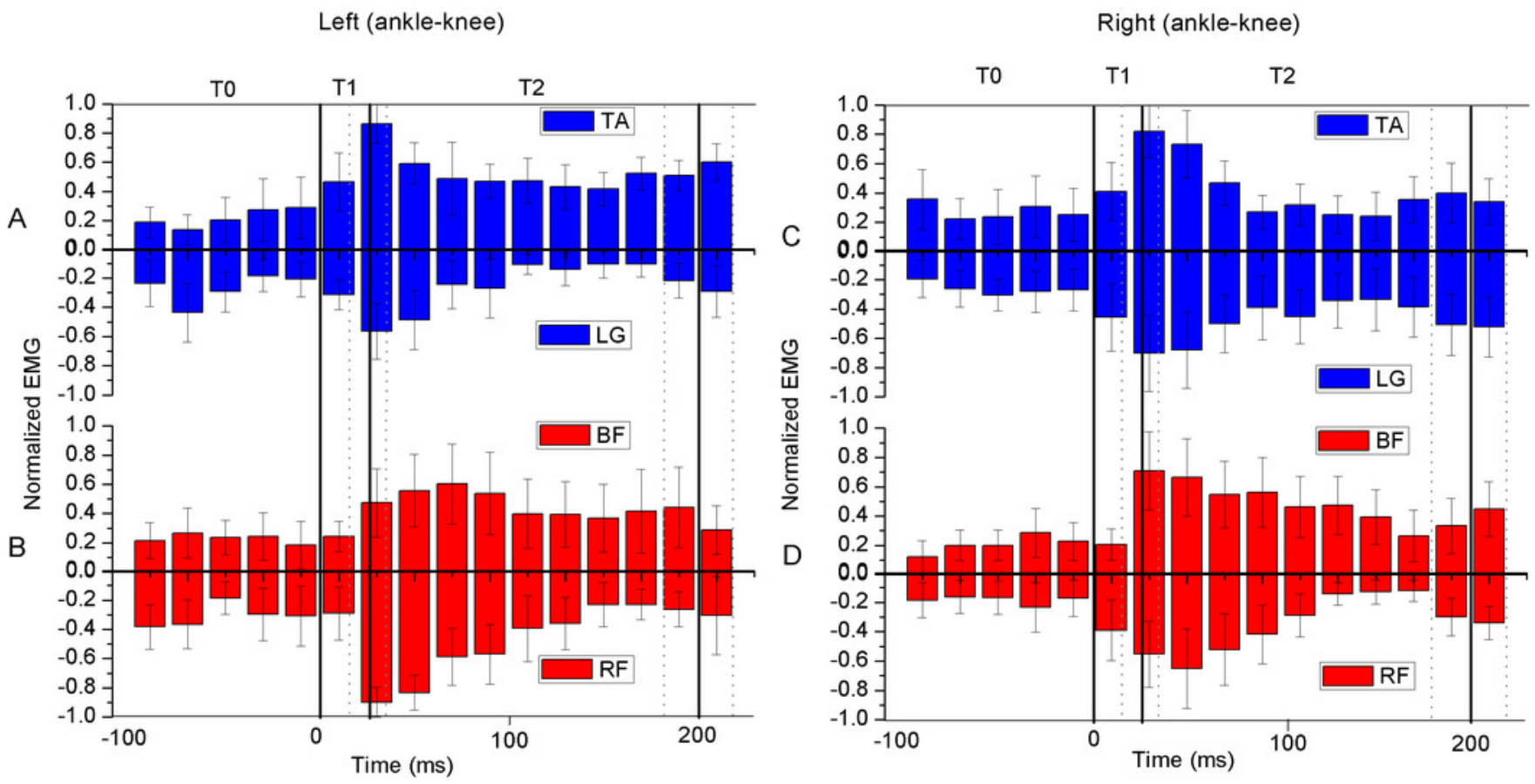


\section{Figure 6}

The angles $(A, B)$, angular velocities $(C, D)$ and torques $(E, F)$ of lower extremity joints for one of the gymnasts during backward somersault landing $(n=1)$.

The skeleton models showed four body postures of the landing (100 ms prior touchdown, touchdown, peak vertical ground reaction force (vGRF), and vGRF equal to body weight, respectively). The whole landing process was divided into three phases by solid vertical lines. TO: The pre-activation phase was defined as 100 ms preceding ground contact; T1: initial impact-phase, from the first touchdown to the peak VGRF; T2: terminal impact-phase, from the peak VGRF to the VGRF equaling to body weight. 


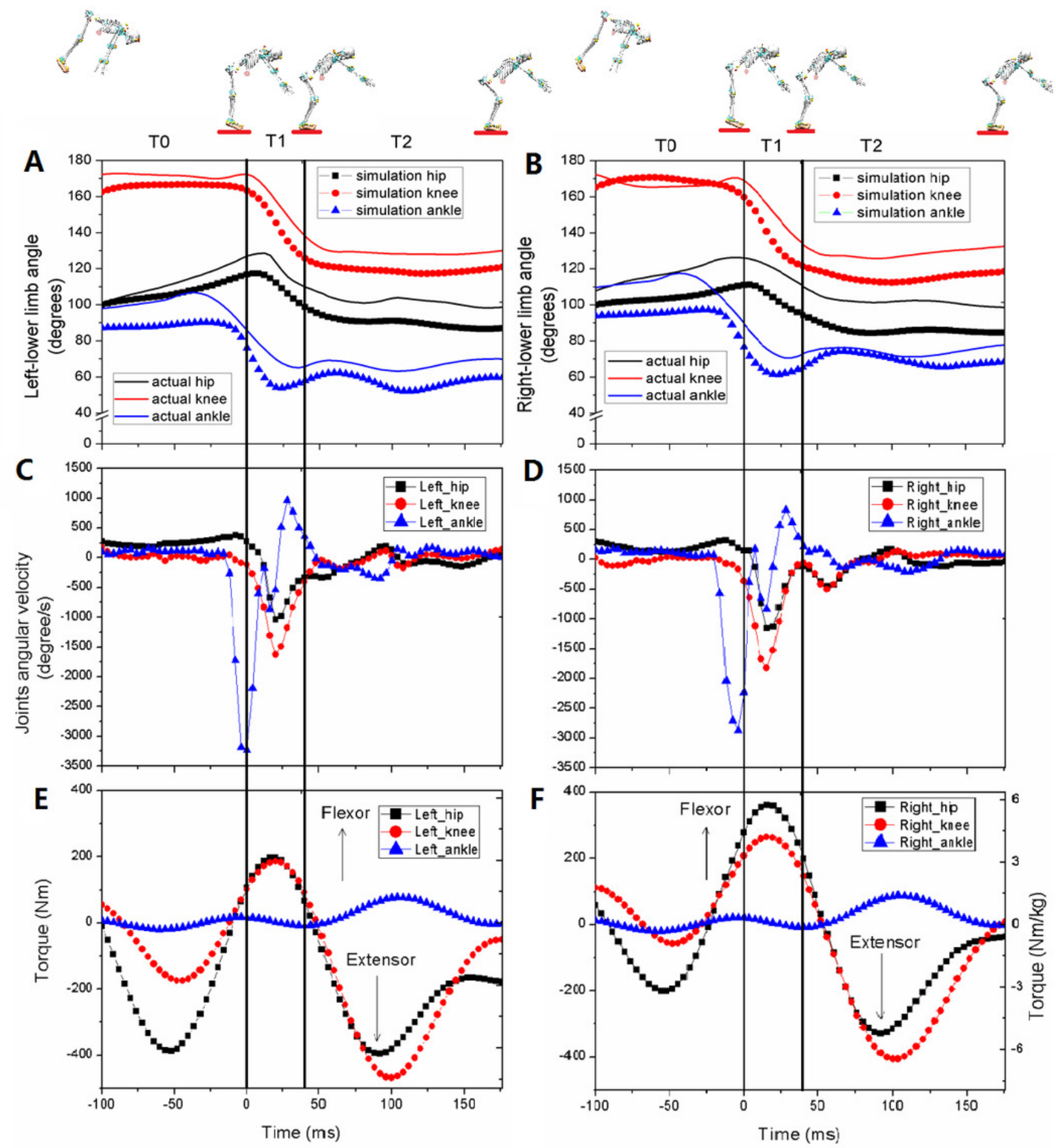

